\title{
Making One's Way Through: Safe Entry Zones to the Brainstem
}

\author{
Puac P1, Sinclair $\mathrm{J}^{1}$, Guarnizo $A^{1}$, Portela de Oliveira $E^{1}$ \\ Ferraciolli $S^{2}$, Glikstein $R^{1}$, Torres $C^{1}$ \\ ${ }^{1}$ University of Ottawa, ON, Canada; ${ }^{2}$ In-RAD-HC-FMSUP, São Paulo, Brazil
}

Disclosure: nothing to disclose.

\section{PURPOSE}

- To describe the imaging landmarks of safe entry zones (SEZs) in the brainstem.

- To illustrate the utility of SEZs in the surgical approach of brainstem lesions.

\section{APPROACH}

- Brainstem lesions represent some of the most challenging entities faced by neurosurgeons.

- SEZs are landmarks of safe but narrow corridors into the brainstem where vital structures are sparse, and no perforating arteries are encountered.

A
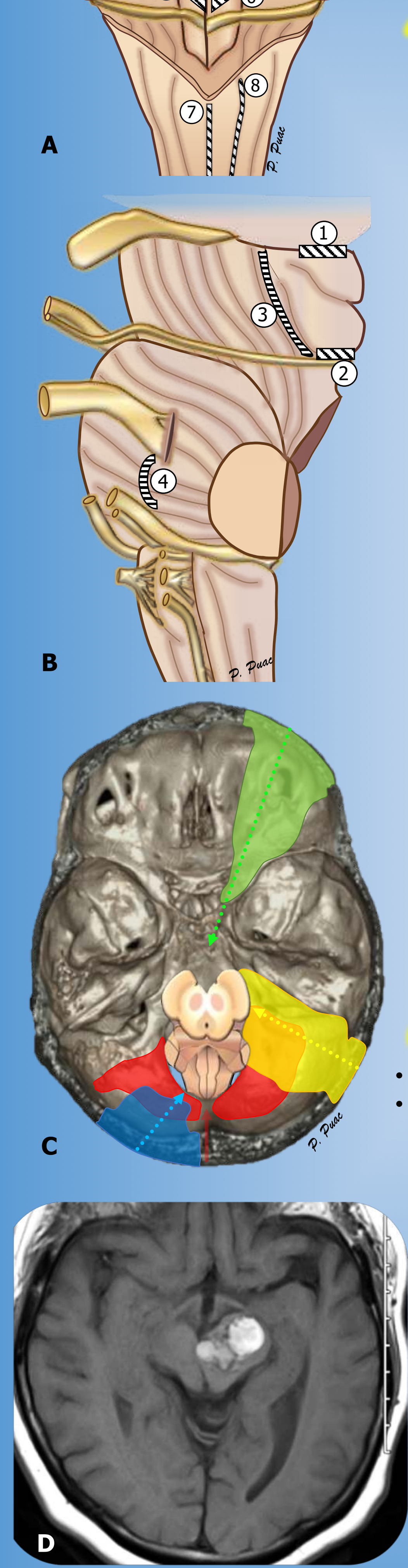

- They are used to approach deep-seated lesions that do not emerge to the pial or ependymal surface of the brainstem.

- Selecting the appropriate entry zone takes into consideration several factors: clinical presentation, lesion location, the shortest and most direct route to the lesion, clinical functions of specific areas, and the degree of comfort of the neurosurgeon with a specific approach.

- Multiple SEZs have been described in the ventral, ventrolateral, and dorsal surfaces of the midbrain, pons, and medulla.

- Large lesions may distort the SEZs, and thus the combination of electrophysiologic mapping, visual microscopic inspection, and neuronavigation ensures the safest entry point.

- The most commonly used and validated SEZs are (Fig. A\&B):

- Midbrain: supracollicular (1), infracollicular (2), and lateral mesencephalic sulcus (LMS,3).

- Pons. peritrigeminal zone (4), suprafacial (5), and infrafacial triangles ( $)$.

- Medulla: posterior median sulcus (7) and posterior intermediate sulcus ( $)$.

- To gain access to these SEZs, different surgical approaches are available being the telovelar/suboccipital $\left(C_{r}\right.$ red area), supracerebellar/infratentorial ( $C_{r}$ blue area), retrosigmoid ( $C_{r}$ yellow area), and orbitozygomatic ( $C_{r}$ green area) approaches the most commonly used.

\section{IMAGING FINDINGS}

Illustrative case. Left midbrain hemorrhagic cavernous malformation (CM). Axial T1-WI ( ) and axial T2-WI (E) MR images demonstrate a heterogenous expansile hemorrhagic cavernoma involving the left cerebral peduncle with associated mass effect on the surrounding structures. The lesion was resected using the LMS safe entry zone ( $E$, $F$, arrows), which is the landmark of a safe corridor between the substantia nigra (SN) $(F)$ and the medial lemniscus (ML) (F). Axial T2-WI of the postoperative MR follow-up (G) shows resection of the $\mathrm{CM}$, with a surgical cavity at the operative site and no evidence of residual disease.

\section{CONCLUSIONS}

- SEZs are landmarks of safe corridors that allow access to deep-seated lesions in the brainstem.

- Radiologists should be aware of these safe corridors and paramount anatomical landmarks in order to aid the neurosurgeons in the preoperative planning of brainstem lesions by offering pertinent imaging guidance and tailored MRI sequences on a case-by-case basis.
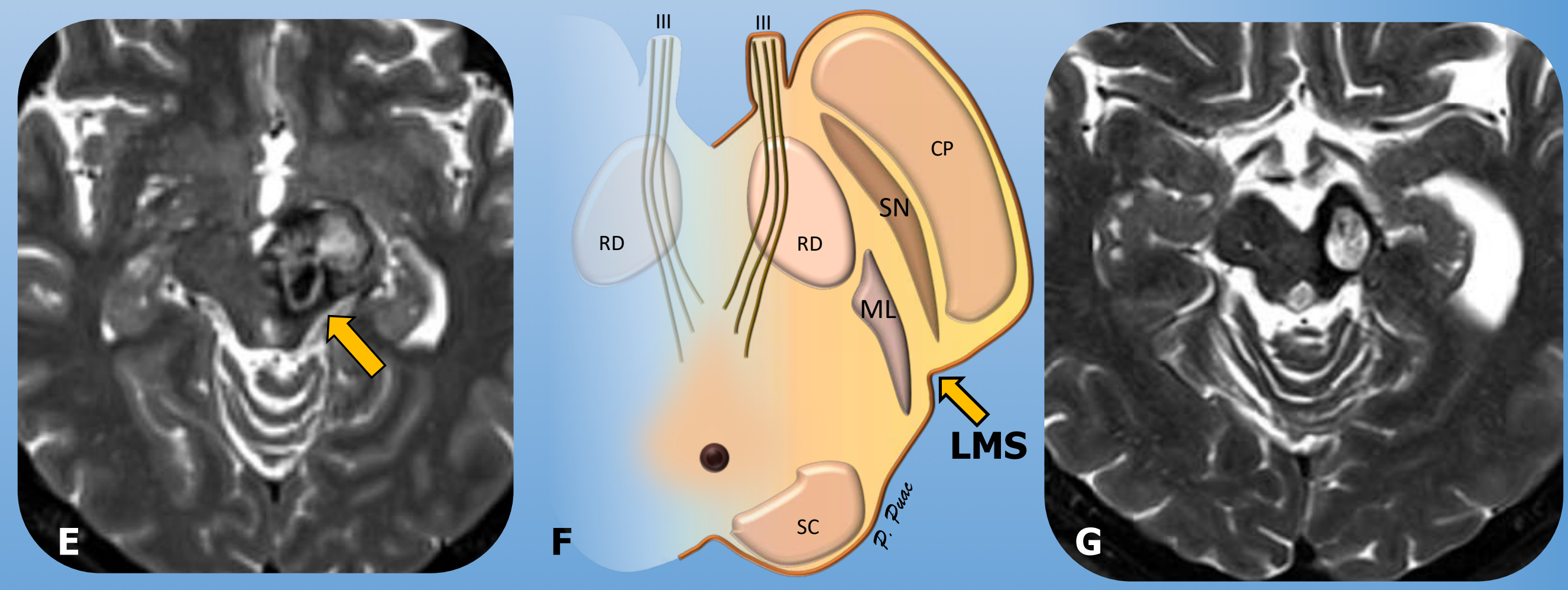

- Recalde RJ, Figueiredo EG, De Oliveira E. Microsurgical anatomy of the safe entry zones on the anterolateral brainstem related to surgical approaches to cavernous malformations. Neurosurgery. 2008;62 - Yang Y, van Niftrik B, Ma X, et al. Analysis of safe entry zones into the brainstem. Neurosurg Rev. 2019;42(3):721-729. doi:10.1007/s10143-019-01081-9

Cavalcanti DD, Preul MC, Kalani MYS, Spetzler RF. Microsurgical anatomy of safe entry zones to the brainstem. J Neurosurg. 2016;124(5):1359-1376. doi:10.3171/2015.4.JNS141945 